\title{
Quantum Dots and Wires to improve Enzymes-Based Electrochemical Bio-sensing
}

\author{
Sandro Carrara*, Cristina Boero, and Giovanni De Micheli \\ EPFL - Swiss Federal Institute of Technology - Lausanne, \\ EPFL IC ISIM LSI1 - INF 338 (Bâtiment INF) Station 14 CH-1015 Lausanne, Switzerland \\ *sandro.carrara@epfl.ch
}

\begin{abstract}
An investigation on nano-structured electrodes to detect different metabolites is proposed in this paper. Three different metabolites are considered: glucose, lactate, and cholesterol. The direct detection of hydrogen peroxide is also considered since it does not involve any enzyme. The metabolites and the peroxide were detected by using screen-printed electrodes modified by using multi-walled carbon nanotubes. In all cases, improvements of orders of magnitude were registered both on detection sensitivity and on detection limit. A close comparison with data recently published in literature has shown the existence of an inverse linear correlation between detection sensitivity and detection limit when sensor performances improve due to nanostructured materials. This inverse linear relationship seems to be a general law as it is here demonstrated for all the considered detections on glucose, lactate, chlesterol, and hydrogen peroxide.
\end{abstract}

Keywords: Quantum Dots, Quantum Wires, Gold Nano-Particles, Carbon Nanotubes, Oxidases, Cytochromes, Electrochemical detection.

\section{Introduction}

Electrochemical bio-sensing is an important branch in biosensing as it enables labelfree detection with important applications to many fields of biomedical diagnostic. For example, gluco-meter sensors present into the market, both for auto-monitoring and for implantable systems [1], are based on glucose oxidase which transforms the glucose into gluconic acid by producing hydrogen peroxide. The hydrogen peroxide oxidation is monitored by means of a current measurement because electrons are directly transferred from the peroxide to the sensor electrode. Similarly, different oxidases may be used to detect lactate, glutamate, cholesterol and other metabolic molecules which may be as relevant as diseases biomarkers [2]. The cholesterol may be also detected by using cytochromes P450 [3]. This is a protein family with more then 3000 different isoforms which may catalyze many different endogenous and exogenous compounds highly relevant for the human metabolism. For example, the

A. Schmid et al. (Eds.): Proceedings of the International Conference Nano-Net 2009,

LNICTS 20, pp.189-199 - Springer, Berlin, 2009 
isoform 11A1 (also called P450scc) catalyzes the cholesterol while other isoforms from the same family catalyze metabolites such as arachidonic acid or thromboxane A2 synthase. The P450 proteins are also important since they may be used to develop label-free drugs screening tools [4]. In case of electrochemical detection based on P450 enzymes, the electrons are directly transferred between the electrode and the cytochrome. For example, the protein $\mathrm{P} 450 \mathrm{scc}$ needs to receive two electrons to transform the cholesterol into pregnenolone as well as the cytochrome 2B4 needs of two electrons in order to transform the benzphetamine into its oxidized form. Both in oxidases and cytochromes, the electron transfer efficiency between electrode and molecules may be enhanced by using nano-structures. It has been demonstrated that the sensitivity in hydrogen peroxide detection is enhanced by two orders of magnitude by using multi-walled carbon nanotubes [5], and one order of magnitude is gained in cholesterol sensitivity for both gold nano-particles [6] and multi-walled carbon nanotubes [7]. Gold nano-particles and carbon nanotubes are nanostructures with quite interesting electrical properties. Nano-particles may be produced by atoms aggregation in solution using gold salts [8]. Particles with different metallic cores may be obtained using other salts containing different metallic ions. Thiol molecules are used to stabilize the particle once is formed. Different particle sizes are obtained by changing the molar ratio between the metallic salts and the thiol stabilizers [9]. The particle sizes are the key parameter to get single-electron trapping inside the particle at room temperature [10]. If the particle size is below $10 \mathrm{~nm}$, then the electrostatic energy of the trapped electrons overcome the thermal excitation which may drop out the electrons from the particle and, therefore, the particle behaves as a quantum-dot. It behaves as a perfect three-dimensional nano-box which may confine quantum carriers. On the other hand, carbon nanotubes may be fabricated by arc-discharge [11] or in a controlled manner by using chemical vapor deposition [12]. It is possible to obtain Single Walled (SW) or Multi Walled (MW) tubes. The lateral sizes are $2 \mathrm{~nm}$ for the SW and $10 \mathrm{~nm}$ for the MW. The nanotubes may present metallic or semimetallic walls. In case of metallic walls, the electron current through the tube is based on ballistic conductivity. In both cases, they present a mean free-path up to $\mu \mathrm{m}$ range at room temperature, which is two orders of magnitude higher than the best macroscopic conductor [13]. This means that a carbon nanotube behaves as a perfect mono-dimensional wire, a perfect quantum-wire which may confine quantum carriers within a lateral size of less then $10 \mathrm{~nm}$ and move them freely for distances of some $\mu \mathrm{m}$.

As we have seen, the amazing electrical properties of both nano-particles and nanotubes have been used to enhance the performances of enzyme based sensors. However, a general conceptual frame enabling us to understand the physics of this enhancement is still lacking in the literature. The understanding of the mechanisms by which nanostructures give such excellent electrochemical performance in sensing is considered a fundamental aspect where research is expected to proceed [14]. Therefore, the aim of this paper is to search a general frame for discussing more relevant and recent results obtained by using nanostructures to improve bio-sensing. The final goal is to investigate general approaches for a theory of electron-transfer enhancement due to quantum-dots and wires in electrochemical detection. 
Quantum Dots and Wires to improve Enzymes-Based Electrochemical Bio-sensing

\section{Materials and Methods}

In this work we have considered oxidases and cytochromes as models of enzymes to detect metabolic molecules. Two different oxidases were considered to sense glucose and lactate while the cytochrome P450 11A1 was considered to sense cholesterol. The direct detection of the hydrogen peroxide was also considered in order to investigate a model where enzymes are not involved. Electrodes were nano-structured by using MW Carbon Nanotubes (MWCNT). Electrochemical measurements were performed on nano-structured electrodes modified by using the considered enzymes.

\subsection{Chemicals}

Accordingly with our previous works [3,6,7], the screen-printed electrodes for P450 investigations were produced on flexible polyvinylchloride sheets from SKK (Denzlingen, Germany) using a DEK 249 screen printer (DEK Ltd., Weymouth, England) by using polyester screens from Steinmann GmbH (Stuttgart, Germany). Screen printing inks (Electrodag PF-410, Electrodag 6037 SS) were obtained from Acheson (Scheemda, Netherlands). Marastar SR 057, from Marabu (Tamm, Germany), was used as insulator ink. In this case, the working electrode is Rhodiumgraphite and it presents an area equal to $20 \mathrm{~mm}^{2}$. Screen-printed electrodes for investigations based on oxidases were instead purchased from Dropsens (model DRP110). In this case the electrode is in graphite and it presents an active area equal to 13 $\mathrm{mm}^{2}$. In both cases, the counter electrode is in graphite and the reference is an $\mathrm{Ag} / \mathrm{AgCl}$ electrode. MWCNT were purchased from MER Corporation (Tucson, AZ) or from Dropsens (Spain). The purchased MWCNT have diameters ranging between 10 and $15 \mathrm{~nm}$ and lengths between 1 and $10 \mu \mathrm{m}$, with 5-20 graphitic layers. The glucose and lactate oxidases were purchased by Sigma-Aldrich as well as hydrogen peroxide and Phosphate Buffer Solution (PBS). The used PBS was a water solution of $137 \mathrm{mM} \mathrm{NaCl}$, with $10 \mathrm{mM}$ Phosphate, and $2.7 \mathrm{mM} \mathrm{KCl}$, at $\mathrm{pH} \mathrm{7.4}$. The $\mathrm{P} 450$ proteins were received from the Institute of Biomedical Chemistry, Russian Academy of Biomedical Science - Moscow (RU).

\subsection{Sensing Electrodes preparation}

We prepared nano-structured screen-printed electrodes by using MWCNT and the probe enzymes. To prepare the MWCNT modified screen-printed electrodes, $100 \mathrm{mg}$ of accurately weighed MWCNT were dispersed in $100 \mathrm{ml}$ of chloroform and ultrasonicated for 1 minute. Finally, the MWNT were deposited by drop casting ( $5 \mu 1$ of the so prepared solution) onto the screen-printed electrodes.

In case of cytochrome P450, the nano-structuring of the screen-printed electrodes was done with only one $5 \mu 1$ drop of MWCNT in an electrode surface of $20 \mathrm{~mm}^{2}$. 
They were further functionalized with a $5 \mu$ l solution containing P450 11A1 (196 $\mu \mathrm{M}$ in $50 \mathrm{mM}$ PBS, pH 7.4, $10 \%$ glycerol, $1 \mathrm{M} \mathrm{NaCl}, 0.3 \%$ sodium cholate). The samples were dried overnight at $+4{ }^{\circ} \mathrm{C}$.

In case of oxidases, the working electrode covering by MWCNT was repeated till a total volume of $40 \mu \mathrm{l}$ of MWCNT was deposited onto the electrode surface of $13 \mathrm{~mm}^{2}$ by adding $5 \mu \mathrm{l}$ each step. Then, about $3.5 \mathrm{U} \mathrm{mm}^{-2}$ for both lactate and glucose oxidases were dropped onto the working electrode and stored overnight at $+4{ }^{\circ} \mathrm{C}$. Then, the drop was rinsed out with Milli-Q before use.

\subsection{Electrochemical Measurements}

The electrochemical response of electrodes was investigated by chronoamperometry under aerobic conditions. Electrochemical measurements were acquired by using a Versastat 3 potentiostat (Princeton Applied Technologies). In the case of cholesterol detection the electrodes were covered with $100 \mu \mathrm{L}$ of PBS, or with the same volume of PBS added to proper amount of the target molecule. In the case of the oxidases, the electrode was dipped into a PBS solution with a volume of $25 \mathrm{ml}$ under stirring conditions. A volume of $25 \mu 1$ of the target molecule was added on the solution each step, in order to reach the concentration of interest. The applied potential was -400 $\mathrm{mV}$ vs $\mathrm{Ag} / \mathrm{AgCl}$ in the case of cholesterol and $+550 \mathrm{mV}$ vs $\mathrm{Ag} / \mathrm{AgCl}$ in the case of glucose, lactate and hydrogen peroxide. Chronoamperometries were taken within time windows in the minutes range during successive additions of target molecules in the same amount.

\section{Results and Discussion}

An increased sensitivity was registered in all of our experiments when we deal with nano-structured electrodes. For example, detection sensitivity increases up to 10 times in case of glucose, and up to 75 times in case of hydrogen peroxide with respect to the case of screen-printed electrodes without MWCNT. Similarly, detection with samples at low concentrations was possible only for electrodes with MWCNT. This shows us that the presence of nanostructures onto screen-printed electrodes increases the sensitivity while decreases the detection limit. This phenomenon was clearly observed for all the three considered metabolites (glucose, lactate, cholesterol) and for hydrogen peroxide, too. Both sensitivity and detection limit have shown changes in orders of magnitude when experiments were performed by using carbon nanotubes. A close comparison with other results recently published in literature was further considered in order to verify the generality of this phenomenon. Moreover, both carbon nanotubes and metallic nano-particles were considered as powering materials to investigate the phenomenon as size-, shape-, or material-related. The next following four tables and figures summarize the results of this comparison. Table 1 shows that MWCNT have shown best performances [15] in respect to SW [16] in case of glucose detection. In both cases, the sensitivity was further pushed by embedding nanotubes within a polymeric matrix, but MWCNT show higher sensitivity and lower detection limit (Table 1). 
Quantum Dots and Wires to improve Enzymes-Based Electrochemical Bio-sensing

Table 1. Different values of detection sensitivity and limit registered in our experiments and reported in literature for the detection of glucose by using different nano-structured materials.

\begin{tabular}{cccc}
\hline Methods & $\begin{array}{c}\text { Limit detection } \\
(\mu \mathrm{M})\end{array}$ & $\begin{array}{c}\text { Sensitivity } \\
\left(\mu \mathrm{mM}^{-1} \mathrm{~cm}^{-2}\right)\end{array}$ & Reference \\
\hline MWCNT-Nafion®-GOD & 1,3 & 171,2 & 15 \\
PDDA-SWCNT-GOD & 4 & 63,84 & 16 \\
B. doped larger-MWCNT/GOD & 3,6 & 2,43 & 17 \\
MWCNT/PtNP/CS/MTOS/GOD & 0,4 & 69,9 & 18 \\
Larger-MWCNT-Nafion®-GOD & 4 & 4,7 & 19 \\
Our sensor (MWCNT-GOD) & 12 & 27,7 & Pres. Work \\
\hline
\end{tabular}

The doping of nanotubes with diameter in the range 20-30 $\mathrm{nm}$ does not increase the performances of the final sensors [17]. The combined deposition of platinum nanoparticles and nanotubes in sol-gel matrix does not affect significantly the sensitivity while decreases of one order of magnitude the detection limit [18]. A lower sensitivity was instead observed for larger MWCNT presenting diameters in the range from 40 up to $60 \mathrm{~nm}$ [19] while a quite high sensitivity and low detection limit were observed by using MWCNT with diameter equal to $10 \mathrm{~nm}$ even without the use of a polymeric matrix (the present work). It is interesting to note that all the reported data follow an inverse relationship between the reached sensitivities and the detection limits, as shown in Figure 1. It shows that different nano-structuring by using single or multiwalled nanotubes, doped or un-doped, embedded or not into polymeric matrix, codeposited with metallic nano-particles, results in different detection sensitivities and detection limits for the final sensor.

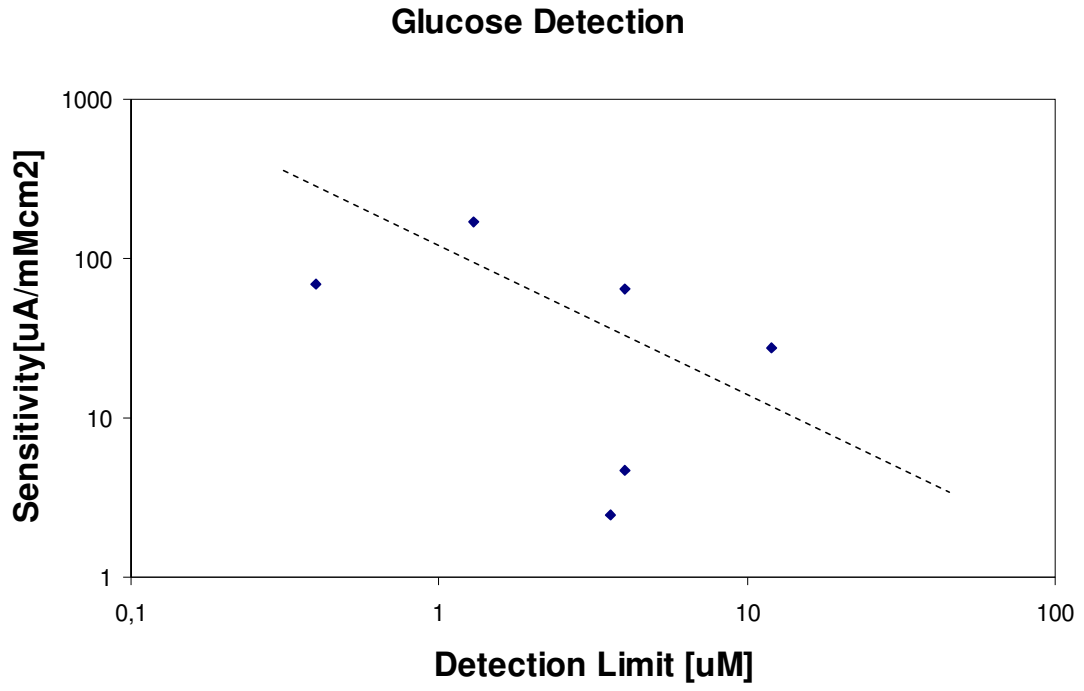

Fig. 1. Detection limits and sensitivities of glucose detectors fabricated by using different nanostructured electrodes. Data are reported in Table 1. The graph in logarithmic scale clearly shows an inverse linear relationship. 
They were further functionalized with a $5 \mu$ l solution containing P450 11A1 (196 $\mu \mathrm{M}$ in $50 \mathrm{mM}$ PBS, pH 7.4, $10 \%$ glycerol, $1 \mathrm{M} \mathrm{NaCl}, 0.3 \%$ sodium cholate). The samples were dried overnight at $+4{ }^{\circ} \mathrm{C}$.

In case of oxidases, the working electrode covering by MWCNT was repeated till a total volume of $40 \mu \mathrm{l}$ of MWCNT was deposited onto the electrode surface of $13 \mathrm{~mm}^{2}$ by adding $5 \mu \mathrm{l}$ each step. Then, about $3.5 \mathrm{U} \mathrm{mm}^{-2}$ for both lactate and glucose oxidases were dropped onto the working electrode and stored overnight at $+4{ }^{\circ} \mathrm{C}$. Then, the drop was rinsed out with Milli-Q before use.

\subsection{Electrochemical Measurements}

The electrochemical response of electrodes was investigated by chronoamperometry under aerobic conditions. Electrochemical measurements were acquired by using a Versastat 3 potentiostat (Princeton Applied Technologies). In the case of cholesterol detection the electrodes were covered with $100 \mu \mathrm{L}$ of PBS, or with the same volume of PBS added to proper amount of the target molecule. In the case of the oxidases, the electrode was dipped into a PBS solution with a volume of $25 \mathrm{ml}$ under stirring conditions. A volume of $25 \mu 1$ of the target molecule was added on the solution each step, in order to reach the concentration of interest. The applied potential was -400 $\mathrm{mV}$ vs $\mathrm{Ag} / \mathrm{AgCl}$ in the case of cholesterol and $+550 \mathrm{mV}$ vs $\mathrm{Ag} / \mathrm{AgCl}$ in the case of glucose, lactate and hydrogen peroxide. Chronoamperometries were taken within time windows in the minutes range during successive additions of target molecules in the same amount.

\section{Results and Discussion}

An increased sensitivity was registered in all of our experiments when we deal with nano-structured electrodes. For example, detection sensitivity increases up to 10 times in case of glucose, and up to 75 times in case of hydrogen peroxide with respect to the case of screen-printed electrodes without MWCNT. Similarly, detection with samples at low concentrations was possible only for electrodes with MWCNT. This shows us that the presence of nanostructures onto screen-printed electrodes increases the sensitivity while decreases the detection limit. This phenomenon was clearly observed for all the three considered metabolites (glucose, lactate, cholesterol) and for hydrogen peroxide, too. Both sensitivity and detection limit have shown changes in orders of magnitude when experiments were performed by using carbon nanotubes. A close comparison with other results recently published in literature was further considered in order to verify the generality of this phenomenon. Moreover, both carbon nanotubes and metallic nano-particles were considered as powering materials to investigate the phenomenon as size-, shape-, or material-related. The next following four tables and figures summarize the results of this comparison. Table 1 shows that MWCNT have shown best performances [15] in respect to SW [16] in case of glucose detection. In both cases, the sensitivity was further pushed by embedding nanotubes within a polymeric matrix, but MWCNT show higher sensitivity and lower detection limit (Table 1). 
Quantum Dots and Wires to improve Enzymes-Based Electrochemical Bio-sensing

Table 2. Different values of detection sensitivity and limit registered in our experiments and reported in literature for the detection of lactate by using different nano-structured materials.

\begin{tabular}{cccc}
\hline Methods & $\begin{array}{c}\text { Limit detection } \\
(\mu \mathrm{M})\end{array}$ & $\begin{array}{c}\text { Sensitivity } \\
\left(\mu \mathrm{A} \mathrm{mM}^{-1} \mathrm{~cm}^{-2}\right)\end{array}$ & Reference \\
\hline Au-Nafion@-TNTs-LOD & 200 & 0,24 & 22 \\
MWCNT-chitosan-LDH & 0,76 & 8,3 & 21 \\
chitosan/PVI/MWCNT/LOD & 5 & 19,7 & 20 \\
sol-gel/MWCNT/LOD & 0,8 & 2,097 & 23 \\
Our sensor (MWCNT-LOD) & 10 & 32,4 & Pres. work \\
\hline
\end{tabular}

However, if the electrodes nano-structuring is efficient enough, then the sensitivity will increase while the detection limit will decrease and the related changes will follow an inverse linear relationship. This phenomenon is quite general, since we observed the same trend in lactate detection, as clearly showed in Figure 2. Even in this case, an inverse linear relationship correlates the detection sensitivity and the detection limit. Table 2 summarizes the values registered in our experiments compared to similar results recently published on lactate detection. Even in that case, the best results were obtained by using the lactate oxidase onto MWCNT alone (the present work) and embedded in a polyvinyl matrix [20] while the lactate dehydrogenase seems to be lesser efficient even when deposited onto carbon nanotubes [21]. Hydrogen titanate nanotubes have not shown performances as good as those of carbon nanotubes [22], neither the use of a bit larger carbon nanotubes with diameter equal to $15 \mathrm{~nm}$ [23].

\section{Lactate Detection}

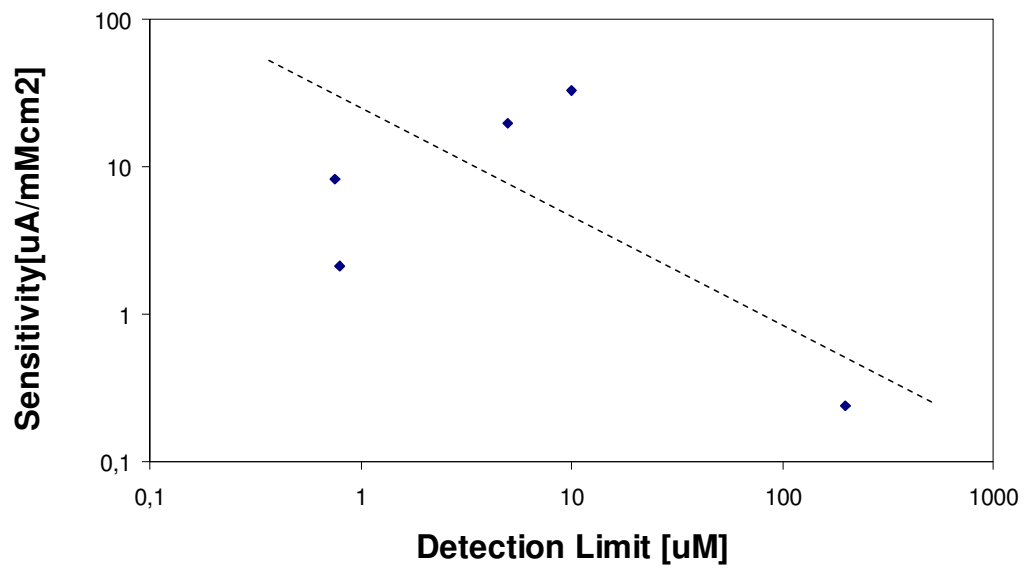

Fig. 2. Detection limits and sensitivities of lactate detectors fabricated by using different nanostructured electrodes. Data are reported in Table 2. The graph in logarithmic scale clearly shows an inverse linear relationship. 
Table 2. Different values of detection sensitivity and limit registered in our experiments and reported in literature for the detection of lactate by using different nano-structured materials.

\begin{tabular}{cccc}
\hline Methods & $\begin{array}{c}\text { Limit detection } \\
(\mu \mathrm{M})\end{array}$ & $\begin{array}{c}\text { Sensitivity } \\
\left(\mu \mathrm{Am}^{-1} \mathrm{~cm}^{-2}\right)\end{array}$ & Reference \\
\hline Au-Nafion®-TNTs-LOD & 200 & 0,24 & 22 \\
MWCNT-chitosan-LDH & 0,76 & 8,3 & 21 \\
chitosan/PVI/MWCNT/LOD & 5 & 19,7 & 20 \\
sol-gel/MWCNT/LOD & 0,8 & 2,097 & 23 \\
Our sensor (MWCNT-LOD) & 10 & 32,4 & Pres. work \\
\hline
\end{tabular}

However, if the electrodes nano-structuring is efficient enough, then the sensitivity will increase while the detection limit will decrease and the related changes will follow an inverse linear relationship. This phenomenon is quite general, since we observed the same trend in lactate detection, as clearly showed in Figure 2. Even in this case, an inverse linear relationship correlates the detection sensitivity and the detection limit. Table 2 summarizes the values registered in our experiments compared to similar results recently published on lactate detection. Even in that case, the best results were obtained by using the lactate oxidase onto MWCNT alone (the present work) and embedded in a polyvinyl matrix [20] while the lactate dehydrogenase seems to be lesser efficient even when deposited onto carbon nanotubes [21]. Hydrogen titanate nanotubes have not shown performances as good as those of carbon nanotubes [22], neither the use of a bit larger carbon nanotubes with diameter equal to $15 \mathrm{~nm}$ [23].

\section{Lactate Detection}

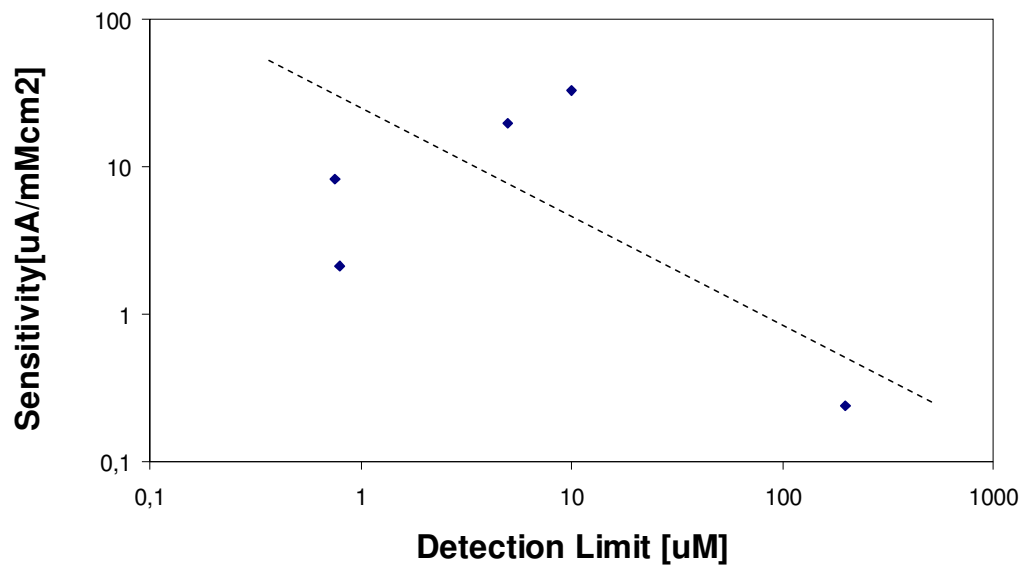

Fig. 2. Detection limits and sensitivities of lactate detectors fabricated by using different nanostructured electrodes. Data are reported in Table 2. The graph in logarithmic scale clearly shows an inverse linear relationship. 
Quantum Dots and Wires to improve Enzymes-Based Electrochemical Bio-sensing

Table 4 summarizes results from cholesterol detection both by using the cholesterol oxidase and the cytochrome P450 11A1 (also called P450scc). The electrons are directly released to the electrode from the proteins in case of the cytochrome. Table 4 shows not high sensitivities for P450 embedded in a molecular matrix containing riboflavine as electron-transfer mediator [3] and for cholesterol oxidase immobilized onto MWCNT [26]. The sensitivity improves of one order of magnitude and detection limit of two orders when carbon nanotubes are used together with platinum nano-particles [27] or with Prussian Blue as electron-transfer mediator [28]. However, the sensitivity gains another order of magnitude when cholesterol is detected by using the cytochrome P450 and gold nano-particles [6] or MWCNT [7]. Also in this case, the reported data clearly indicate an inverse linear relationship between detection sensitivity and limit with nano-structured electrodes both in case of oxidase and cytochrome.

\section{Conclusions}

Enzymes based detection of different metabolites (glucose, lactate, and cholesterol) was considered in this paper. In all cases, detection improvements due to electrodes nano-structuring were investigated. The detection of hydrogen peroxide was also considered in order to investigate a case where no proteins are involved. In all the cases, improvements were registered by structuring the screen-printed electrodes with Multi Walled Carbon Nanotubes. By comparing the obtained results with others recently published in literature, an inverse linear correlation-law between detection sensitivity and detection limit of the sensor upon different nano-structuring was discovered. Thus, a general law is here shown relaying that sensitivity and limit in detection are changing with a linear relation when electrodes nano-structuring improves sensor performances. This means that we found a general law for a deep understanding of a general theory in bio-sensing improvements due to quantum-dots and quantum-wires. Further work will be focused on designing an equations set to describe where and how quantum-structures affect the electrochemical bio-sensing.

Acknowledgments. Victoria Shumyantseva is acknowledged for providing P450 based materials, and for her precious support in electrochemistry investigations. Manas Jain is acknowledged for help in manuscript revision. The research has been partially funded by the EPFL' Integrated Center SI project titled "Enabling Nano-BioChip Technologies for Sensing Applications", and by the Sino-Swiss cooperation project titled "Nano-structured Bio-Chip development for Stem Cells Monitoring", project code IZLCZ2 123967.

\section{References}

1. Klonoff, D.C.: Continuous glucose monitoring - Roadmap for 21 st century diabetes therapy. Diabetes Care 28, 1231--1239 (2005)

2. Boero, C., Carrara S., De Micheli, G.: Sensitivity Enhancement by Carbon Nanotubes: Applications to Stem Cell Cultures Monitoring. In: $5^{\text {th }}$ International Conference on Ph.D. Research in Microelectronics \& Electronics, Cork, Ireland (2009) 
3. Shumyantseva, V., De Luca, G., Bulko, T., Carrara, S., Nicolini, C., Usanov, S.A., Archakov, A.: Cholesterol amperometric biosensor based on cytochrome P450scc. Biosensors and Bioelectronics 19, 971--976 (2004)

4. Joseph, S., Rusling, J.F., Lvov, Y.M., Friedberg, T., Fuhr, U.: An amperometric biosensor with human CYP3A4 as a novel drug screening tool. Biochem. Pharmacol. 65, 1817--1826 (2003)

5. Cui, X., Li, C.M., Zang, J., Yu, S.: Highly sensitive lactate biosensor by engineering chitosan/PVI-Os/CNT/LOD network nanocomposite. Biosensors and Bioelectronics 22, 3288--3292 (2007)

6. Shumyantseva, V.V., Carrara, S., Bavastrello, V., Riley, D.J., Bulko, T.V., Skryabin, K.G., Archakov, A.I., Nicolini, C.: Direct electron transfer between cytochrome P450scc and gold nanoparticles on screen-printed rhodium-graphite electrodes. Biosensors and Bioelectronics 21, 217--222 (2005).

7. Carrara, S., Shumyantseva, V.V., Archakov, A.I., Samorì, B.: Screen-printed electrodes based on carbon nanotubes and cytochrome P450scc for highly sensitive cholesterol biosensors. Biosensors and Bioelectronics 24 148--150 (2008)

8. Brust, M., Walker, M., Bethell, D., Schiffrin, D.J., Whyman, R.: Synthesis of thiolderivatized gold nanoparticles in a 2-phase liquid-liquid system. J. Chem. Soc. Chem. Commun. 801--802 (1994)

9. Hostetler, M.J., Wingate, J.E., Zhong, C.J., Harris, J.E., Vachet, R.W., Clark, M.R., Londono, J.D., Green, S.J., Stokes, J.J., Wignall, G.D., Glish, G.L., Porter, M.D., Evans, N.D., Murray, R.W.: Alkanethiolate gold cluster molecules with core diameters from 1.5 to $5.2 \mathrm{~nm}$ : Core and monolayer properties as a function of core size. Langmuir 14, 17--30 (1998)

10. Carrara, S., Erokhin, V., Facci, P., Nicolini, C.: On the role of nanoparticle sizes in monoelectron conductivity. In: Fendler, J., Dékány, I. (eds.) Nanoparticle in Solid and Solutions. Kluwer publisher, Dordrecht (NL). NATO ASI Series 3. High Technology - vol. 18, pp. 497--503 (1996)

11. H. H. Kim, H. J. Kim, Preparation of carbon nanotubes by DC arc discharge process under reduced pressure in an air atmosphere, Materials Science and Engineering, B133, ( 2006) 241-244

12. C. He, N. Zhao, Y. Han, J. Li, C. Shi, X. Du, Study of aluminum powder as transition metal catalyst carrier for CVD synthesis of carbon nanotubes, Materials Science and Engineering: A 441( 2006) 266--270

13. H. J. Li, W. G. Lu, J. J. Li, X. D. Bai, and C. Z. Gu, Multichannel Ballistic Transport in Multiwall Carbon Nanotubes, Phys. Rev. Lett., 95 (2005) 086601-1--086601-4

14. Gooding, J.J.: Nanostructuring electrodes with carbon nanotubes: A review on electrochemistry and applications for sensing. Electrochim. Acta 50, 3049--3060 (2005).

15. Rahman, M.M., Umar, A., Sawada, K.: Development of amperometric glucose biosensor based on glucose oxidase co-immobilized with multi-walled carbon nanotubes at low potential. Sensors and Actuators B 137, 327--333 (2009)

16. Wang, Y., Wang, X., Wu, B., Zhao, Z., Yin, F., Li, S., Qin, X., Chen, Q.: Dispersion of single-walled carbon nanotubes in poly(diallyldimethylammonium chloride) for preparation of a glucose biosensor. Sensors and Actuators B 130, 809-815 (2008)

17. Chen, X., Chen, J., Deng, C., Xiao, C., Yang, Y., Nie, Z., Yao, S.: Amperometric glucose biosensor based on boron-doped carbon nanotubes modified electrode. Talanta 76, 763--767 (2008)

18. Kang, X., Mai, Z., Zou, X., Cai, P., Mo, J.: Glucose biosensors based on platinum nanoparticles-deposited carbon nanotubes in sol-gel chitosan/silica hybrid. Talanta 74, 879$-886(2008)$

19. Tsai, Y.C., Li, S.C., Chen, J.M.: Cast Thin Film Biosensor Design Based on a Nafion Backbone, a Multiwalled Carbon Nanotube Conduit, and a Glucose Oxidase Function. Langmuir 21, 3653--3658 (2005) 
Quantum Dots and Wires to improve Enzymes-Based Electrochemical Bio-sensing

20. Cui, X., Li, C.M., Zang, J., Yu, S.: Highly sensitive lactate biosensor by engineering chitosan/PVI-Os/CNT/LOD network nanocomposite, Biosensors and Bioelectronics 22, 3288--3292 (2007)

21. Tsai, Y.C., Chen, S.Y., Liaw, H.W.: Immobilization of lactate dehydrogenase within multiwalled carbon nanotube-chitosan nanocomposite for application to lactate biosensors. Sensor. Actuat. B 125, 474--481 (2007)

22. Yang, M., Wang, J., Li, H., Zheng, J.G., Wu, N.N.: A lactate electrochemical biosensor with a titanate nanotube as direct electron transfer promoter. Nanotechnoloy 19, 075502 (2008)

23. Huang, J., Song, Z., Li, J., Yang, Y., Shi, H., Wu, B., Anzai, J.I., Osa, T., Chen, Q.: A highly-sensitive 1-lactate biosensor based on sol-gel film combined with multi-walled carbon nanotubes (MWCNTs) modified electrode. Mat. Sci. Eng. C 27, 29--34 (2007)

24. Guzmán, C., Orozco, G., Verde, Y., Jiménez, S., Godínez, L.A., Juaristi, E., Bustos, E.: Hydrogen peroxide sensor based on modified vitreous carbon with multiwall carbon nanotubes and composites of Pt nanoparticles-dopamine. Electrochim. Acta 54, 1728--1732 (2009)

25. Pang, X., He, D., Luo, S., Cai, Q.: An amperometric glucose biosensor fabricated with Pt nanoparticle-decorated carbon nanotubes/TiO2 nanotube arrays composite. Sensors and Actuators B 137, 134--138 (2009)

26. Li, G., Liao, J.M., Hu, G.Q., Ma, N.Z., Wu, P.J.: Study of carbon nanotube modified biosensor for monitoring total cholesterol in blood. Biosensors and Bioelectronics 20, 2140$-2144(2005)$

27. Yang, M., Yang, Y., Yang, H., Shen, G., Yu, R.: Layer-by-layer self-assembled multilayer films of carbon nanotubes and platinum nanoparticles with polyelectrolyte for the fabrication of biosensors. Biomaterials 27, 246--255 (2006)

28. Tan, X., Li, M., Cai, P., Luo, L., Zou, X.: An amperometric cholesterol biosensor based on multiwalled carbon nanotubes and organically modified sol-gel/chitosan hybrid composite film. Anal. Biochem. 337, 111--120 (2005) 